Pacific Journal of Mathematics

ON WITT'S THEOREM FOR UNIMODULAR QUADRATIC 


\title{
ON WITT'S THEOREM FOR UNIMODULAR QUADRATIC FORMS
}

\author{
D. G. JAMES
}

\begin{abstract}
In this paper we give an integral generalization of Witt's theorem for quadratic forms. If $J$ and $K$ are sublattices of a unimodular lattice $L$, we investigate conditions under which an isometry from $J$ to $K$ will extend to an isometry of $L$.
\end{abstract}

Let $L$ be a free $Z$-module (that is a lattice) of finite rank and $\Phi: L \times L \rightarrow Z$ a unimodular symmetric bilinear form on $L$. We denote $\Phi(\alpha, \beta)$ by $\alpha \cdot \beta$, so that $\alpha \cdot \beta=\beta \cdot \alpha$. A bijective linear mapping $\varphi: J \rightarrow K$, where $J$ and $K$ are sublattices of $L$, is called an isometry if $\varphi(\alpha) \cdot \varphi(\beta)=\alpha \cdot \beta$ for $\alpha, \beta \in J$. Witt's theorem concerns the extension of such an isometry to an isometry of $L$ (onto $L$ ). The set of isometries of $L$ form the orthogonal group $O(L, Z)$ of $L$.

Vectors $\alpha$ and $\beta$ in $L$ are called orthogonal if $\alpha \cdot \beta=0 ; \alpha^{2}$ denotes $\alpha \cdot \alpha$, the norm of $\alpha$. Any nonzero vector $\alpha \in L$ may be written as $\alpha=d \beta$ with $\beta \in L, d \in Z$ maximal. If $d=1, \alpha$ is called primitive; $d$ is the divisor of $\alpha$. It is clear that an isometry $\varphi$ of $L$ must leave invariant the divisors of all vectors; that is, $\alpha$ and $\varphi(\alpha)$ have the same divisor.

A sublattice $U$ of $L$ is called primitive if all the vectors of $U$ which are "primitive in $U$ " are also "primitive in $L$ ". In particular the basis vectors of $U$ must be primitive (in $L$ ). In considering the extension of an isometry $\varphi: J \rightarrow K$ to an isometry of $L$, it clearly suffices to consider the case where $J$ and $K$ are primitive sublattices.

A primitive vector $\alpha \in L$ is called characteristic if $\alpha \cdot \beta \equiv \beta^{2}$ $(\bmod 2)$ for all $\beta \in L$. Again it is clear that an isometry must map a characteristic vector into a characteristic vector.

Let $r(L)$ and $s(L)$ denote the rank and signature of $L$. Then we shall prove the following.

THEOREM. Let $\varphi: J \rightarrow K$ be an isometry between the primitive sublattices $J$ and $K$ of $L$, where

$$
r(L)-|s(L)| \geqq 2(r(J)+1) .
$$

Then $\varphi$ extends to an isometry of $L$ if and only if:

$\alpha$ a characteristic vector $\Leftrightarrow \varphi(\alpha)$ a characteristic vector (for each $\alpha$ in $J)$.

This result is a generalization of Wall [1]; in fact we shall use 
similar arguments and many of the results contained in Wall's paper.

1. Let $\left\langle\alpha_{1}, \alpha_{2}, \cdots, \alpha_{m}\right\rangle$ denote the lattice spanned by the vectors $\alpha_{1}, \alpha_{2}, \cdots, \alpha_{m}$. If $L$ is the orthogonal direct sum of the sublattices $U$ and $V$ we write $L=U \oplus V$. In this case we say $U$ (or $V$ ) splits $L$. $U^{\perp}$ will denote the orthogonal complement of $U$.

We show first how to reduce the proof to the case where $s(L)=0$. Let $s(L)=s$. We consider the case $s>0(s<0$ is similar). Enlarge the lattice $L$ to

$$
L^{\prime}=L \oplus\left\langle\zeta_{1}\right\rangle \oplus \cdots \oplus\left\langle\zeta_{s}\right\rangle
$$

where $\zeta_{i}^{2}=-1,1 \leqq i \leqq s$, so that $s\left(L^{\prime}\right)=0$. Let

$$
J^{\prime}=J \oplus\left\langle\zeta_{1}\right\rangle \oplus \cdots \oplus\left\langle\zeta_{s}\right\rangle
$$

and

$$
K^{\prime}=K \oplus\left\langle\zeta_{1}\right\rangle \oplus \cdots \oplus\left\langle\zeta_{s}\right\rangle
$$

$J^{\prime}$ and $K^{\prime}$ are primitive sublattices of $L^{\prime}$. Furthermore if $L$ satisfies (1)

$$
r\left(L^{\prime}\right)-s\left(L^{\prime}\right)=r(L)+s \geqq 2\left(r\left(J^{\prime}\right)+1\right) .
$$

Also, extending $\varphi$ to $J^{\prime}$ by $\varphi\left(\zeta_{i}\right)=\zeta_{i}$, we see immediately that $\alpha \in J^{\prime}$ is characteristic if and only if $\phi(\alpha) \in K^{\prime}$ is characteristic. (Notice that if $\alpha \in L^{\prime}$ is characteristic, all the coefficients of the $\zeta_{i}$ in $\alpha$ must be odd.) If, therefore, we establish the theorem when the signature is zero, we know $\varphi$ extends to an isometry of $L^{\prime}$. Restricting back to $L$ will establish the general result.

From now on we assume $s(L)=0$. Let $H$ denote a hyperbolic plane of the form $\langle\lambda, \mu\rangle$ where $\lambda^{2}=\mu^{2}=0$ and $\lambda \cdot \mu=1$; and let $I$ denote a sublattice of the form $\langle\xi, \rho\rangle=\langle\xi\rangle \oplus\langle\xi-\rho\rangle$ where $\xi^{2}=$ $\xi \cdot \rho=1$ and $\rho^{2}=0$. Then it is well known that any unimodular lattice of zero signature is either an orthogonal direct sum of $H^{\prime}$ s (if improper) or an orthogonal direct sum of $I^{\prime} \mathrm{s}$ (if proper); see Wall [1, Th. 5]. We might also mention that if $L$ is improper there are no primitive characteristic vectors.

Before proving the theorem we give an example to show the necessity of the restriction (1) we have placed on the ranks of $L$ and $J$.

EXAMPLE. Let

$$
L=H_{1} \oplus H_{2} \oplus \cdots \oplus H_{n}
$$

where $H_{i}=\left\langle\lambda_{i}, \mu_{i}\right\rangle, 1 \leqq i \leqq n$. Take 


$$
J=\left\langle\lambda_{1}, \cdots, \lambda_{n-1}, \lambda_{n}+u v \mu_{n}\right\rangle
$$

and

$$
K=\left\langle\lambda_{1}, \cdots, \lambda_{n-1}, u \lambda_{n}+v \mu_{n}\right\rangle
$$

where $u$ and $v$ are integers $(\neq \pm 1)$ such that $(u, v)=1$. We shall show that the isometry $\varphi: J \rightarrow K$ defined by

$$
\begin{gathered}
\varphi\left(\lambda_{i}\right)=\lambda_{i}, \quad 1 \leqq i \leqq n-1, \\
\varphi\left(\lambda_{n}+u v \mu_{n}\right)=u \lambda_{n}+v \mu_{n},
\end{gathered}
$$

does not extend to an isometry of $L$. For if it did, (2) and the conditions $\lambda_{i} \cdot \varphi\left(\mu_{n}\right)=\varphi\left(\lambda_{i}\right) \cdot \varphi\left(\mu_{n}\right)=\lambda_{i} \cdot \mu_{n}=0,1 \leqq i \leqq n-1$, would force

$$
\varphi\left(\mu_{n}\right)=x_{1} \lambda_{1}+x_{2} \lambda_{2}+\cdots+x_{n-1} \lambda_{n-1}+x \lambda_{n}+y \mu_{n}
$$

and

$$
\begin{aligned}
\varphi\left(\lambda_{n}\right)= & -u v x_{1} \lambda_{1}-u v x_{2} \lambda_{2}-\cdots-u v x_{n-1} \lambda_{n-1} \\
& +u(1-v x) \lambda_{n}+v(1-u y) \mu_{n}
\end{aligned}
$$

for some integers $x_{1}, \cdots, x_{n-1}, x, y$ as yet undetermined. But $\varphi\left(\mu_{n}\right)^{2}=$ $\mu_{n}^{2}=0$ implies that $x y=0$; while $\varphi\left(\lambda_{n}\right) \cdot \varphi\left(\mu_{n}\right)=1$ implies $x v+y u=1$. These two conditions are incompatible with our choice $u, v \neq \pm 1$. Thus we need, at least, $r(L)>2 r(J)$.

We shall now proceed with the proof of the theorem. There will be three stages in the proof.

(i) First we establish the result when $L$ is improper. In this case there are no characteristic vectors to consider.

(ii) Secondly, we consider $L$ proper, but with $J$ and $K$ containing no characteristic vectors.

(iii) Finally, we treat the general proper case.

Notation. The following notation will be used for an isometry. Let

$$
L=\left\langle\alpha_{1}, \alpha_{2}, \cdots, \alpha_{m}\right\rangle \oplus U=\left\langle\beta_{1}, \beta_{2}, \cdots, \beta_{m}\right\rangle \oplus U
$$

where $\alpha_{i} \cdot \alpha_{j}=\beta_{i} \cdot \beta_{j}, 1 \leqq i, j \leqq m$. Then

$$
\theta:\left\langle\alpha_{1}, \alpha_{2}, \cdots, \alpha_{m}\right\rangle \rightarrow\left\langle\beta_{1}, \beta_{2}, \cdots, \beta_{m}\right\rangle
$$

is the isometry of $L$ defined by $\theta\left(\alpha_{i}\right)=\beta_{i}, 1 \leqq i \leqq m$, with $\theta$ restricted to $U$ being the identity map.

Many of the isometries will be used repeatedly. We will label them $\theta_{1}, \theta_{2}, \cdots$ as they are defined so that we may refer back to them. 
2. Throughout this section we let $L$ be of the form

$$
L=\left\langle\lambda_{1}, \mu_{1}\right\rangle \oplus \cdots \oplus\left\langle\lambda_{n}, \mu_{n}\right\rangle
$$

where each $\left\langle\lambda_{i}, \mu_{i}\right\rangle$ is a hyperbolic plane. The following lemma follows immediately from Wall [1, Th. 1].

LEMMA 1. Let $r(L) \geqq 4$. For each primitive vector $\alpha \in L$ there exists an isometry $\psi \in o(L, Z)$ such that

$$
\psi(\alpha)=\lambda_{1}+\frac{1}{2} \alpha^{2} \mu_{1}
$$

As a first step in the proof of the theorem we show there exists an isometry $\psi \in o(L, Z)$ such that $\psi(J)=\left\langle\alpha_{1}, \cdots, \alpha_{m}\right\rangle$, where

$$
\left\{\begin{aligned}
\alpha_{1} & =\lambda_{1}+c_{1} \mu_{1} \\
\alpha_{2} & =a_{12} \mu_{1}+\lambda_{2}+c_{2} \mu_{2} \\
& \cdots \cdots \cdots \cdots \\
\alpha_{m} & =a_{1 m} \mu_{1}+a_{2 m} \mu_{2}+\cdots+a_{m-1 m} \mu_{m-1}+\lambda_{m}+c_{m} \mu_{m} .
\end{aligned}\right.
$$

We use induction on $m$. The case $m=1$ is Lemma 1 . Assume now $\alpha_{1}, \alpha_{2}, \cdots, \alpha_{h}$ have been constructed using an isometry $\psi_{1}$; that is $\psi_{1}(J)=\left\langle\alpha_{1}, \cdots, \alpha_{h}, \beta, \gamma, \cdots\right\rangle$. Adding to $\beta$ linear combinations of $\alpha_{1}, \cdots, \alpha_{h}$ (if necessary) we may assume $\beta$ has the form

$$
\beta=\sum_{i=1}^{h} b_{i} \mu_{i}+\sum_{i=h+1}^{n}\left(a_{i} \lambda_{i}+b_{i} \mu_{i}\right) \text {. }
$$

By applying Lemma 1 on $E=\left\langle\lambda_{h+1}, \mu_{h+1}\right\rangle \oplus \cdots \oplus\left\langle\lambda_{n}, \mu_{n}\right\rangle$ to the component of $\beta$ in $E(r(E) \geqq 4$ by (1)), we may assume

$$
\beta=\sum_{i=1}^{h} b_{i} \mu_{i}+a \lambda_{h+1}+b \mu_{h+1} .
$$

If $(a, b)=1$ we may obtain $\alpha_{h+1}$ by using Lemma 1 on the component $a \lambda_{h+1}+b \mu_{h+1}$ in $E$. Otherwise we proceed as follows. We may assume $\beta$ primitive, so that $\left(b_{1}, \cdots, b_{h}, a, b\right)=1$. Apply the isometry (writing $k$ for $h+2$ );

$$
\begin{aligned}
& \theta_{1}:\left\langle\lambda_{1}, \mu_{1}\right\rangle \oplus\left\langle\lambda_{2}, \mu_{2}\right\rangle \oplus \cdots \oplus\left\langle\lambda_{h}, \mu_{h}\right\rangle \oplus\left\langle\lambda_{k}, \mu_{k}\right\rangle \rightarrow \\
& \quad\left\langle\lambda_{1}-c_{1} \mu_{k}, \mu_{1}+\mu_{k}\right\rangle \oplus\left\langle\lambda_{2}-a_{12} \mu_{k}, \mu_{2}\right\rangle \oplus \cdots \oplus\left\langle\lambda_{h}-a_{1 h} \mu_{k}, \mu_{h}\right\rangle \\
& \quad \bigoplus\left\langle\lambda_{k}-\lambda_{1}+c_{1} \mu_{1}+a_{12} \mu_{2}+\cdots+a_{1 h} \mu_{h}+c_{1} \mu_{k}, \mu_{k}\right\rangle .
\end{aligned}
$$

Then, we see, $\theta_{1}\left(\alpha_{i}\right)=\alpha_{i}$ for $1 \leqq i \leqq h$, and $\theta_{1}(\beta)=\beta+b_{1} \mu_{k}$. Applying Lemma 1 to the component of $\theta_{1}(\beta)$ in $E$, namely $a \lambda_{h+1}+b \mu_{h+1}+b_{1} \mu_{k}$, we can transform it back to the form of (4), but now with

$$
\left(b_{2}, b_{3}, \cdots, b_{h}, a, b\right)=1 \text {. }
$$


Repeating this process, this time in $\left\langle\lambda_{1}, \mu_{1}\right\rangle^{\perp}$, we may obtain a new $\beta$ this time with $\left(b_{3}, \cdots, b_{h}, a, b\right)=1$. Ultimately, we obtain a $\beta$ with $(a, b)=1$, so that we may finish by using lemma 1 as before.

It now suffices to prove the theorem with $J=\left\langle\alpha_{1}, \cdots, \alpha_{m}\right\rangle$. We shall prove the theorem by induction on $r(J)$. When $r(J)=1$, the result follows from Wall (our Lemma 1). For the general case we may assume $K$ has the form $\left\langle\alpha_{1}, \cdots, \alpha_{m-1}, \alpha\right\rangle$, with $\varphi: J \rightarrow K$ being the mapping defined by $\varphi\left(\alpha_{i}\right)=\alpha_{i}$ for $1 \geqq i \leqq m-1$, and

$$
\varphi\left(\alpha_{m}\right)=\alpha=\sum_{i=1}^{m-1}\left(x_{i} \lambda_{i}+y_{i} \mu_{i}\right)+u \lambda_{m}+v \mu_{m} .
$$

(It suffices to consider $u \lambda_{m}+v \mu_{m}$ by Lemma 1 ). It remains to find an isometry $\psi \in o(L, Z)$ such that $\psi\left(\alpha_{i}\right)=\alpha_{i}$ for $1 \leqq i \leqq m-1$, and $\psi\left(\alpha_{m}\right)=\alpha$.

We show first that we may take $u=1$. Using Lemma 1 , we may assume $u$ divides $v$. Now $\alpha-\sum_{i=1}^{m-1} x_{i} \alpha_{i}$ is primitive (since $K$ is a primitive lattice), so that

$$
\left(u, z_{m-1}, \cdots, z_{2}, z_{1}\right)=1
$$

where

$$
\left\{\begin{array}{rl}
z_{m-1} & =y_{m-1}-x_{m-1} c_{m-1} \\
& \cdots \cdots \cdots \cdots \cdots \cdots \cdots \cdots \cdots \cdots \cdots \\
z_{2} & =y_{2}-x_{2} c_{2}-x_{3} a_{23}-\cdots \cdots x_{m-1} a_{2 m-1} \\
z_{1} & =y_{1}-x_{1} c_{1}-x_{2} a_{12}-\cdots-x_{m-1} a_{1 m-1}
\end{array} .\right.
$$

We apply the isometry $\theta_{1}$ again, but with $h$ replaced by $m-1$ and $k(=h+2)$ by $m+1$. As before $\theta_{1}\left(\alpha_{i}\right)=\alpha_{i}$ for $1 \leqq i \leqq m-1$, but now

$$
\theta_{1}(\alpha)=\alpha+z_{1} \mu_{m+1} \text {. }
$$

Using Lemma 1 on $u \lambda_{m}+v \mu_{m}+z_{1} \mu_{m+1}$ in $\left\langle\lambda_{m}, \mu_{m}\right\rangle \oplus\left\langle\lambda_{m+1}, \mu_{m+1}\right\rangle$, we may replace $\alpha$ by a new $\alpha$ in which $u$ divides $z_{1}$. By repeating this argument, now in $\left\langle\lambda_{1}, \mu_{1}\right\rangle^{\perp}$, we can get a new $u$ again, this time also dividing $z_{2}$. Eventually, from (6), we may reduce $u$ to 1 .

Finally, we reduce the $x_{1}, \cdots, x_{m-1}$ in (5), in turn to zero. Apply the isometry

$$
\begin{aligned}
\theta_{2}: & \left\langle\lambda_{1}, \mu_{1}\right\rangle \oplus\left\langle\lambda_{2}, \mu_{2}\right\rangle \oplus \cdots \oplus\left\langle\lambda_{m-1}, \mu_{m-1}\right\rangle \oplus\left\langle\lambda_{m}, \mu_{m}\right\rangle \rightarrow \\
& \left\langle\lambda_{1}-x_{1} c_{1} \mu_{m}, \mu_{1}+x_{1} \mu_{m}\right\rangle \oplus\left\langle\lambda_{2}-x_{1} a_{12} \mu_{m}, \mu_{2}\right\rangle \\
& \oplus \cdots \oplus\left\langle\lambda_{m-1}-x_{1} a_{1 m-1} \mu_{m}, \mu_{m-1}\right\rangle \\
& \oplus\left\langle\lambda_{m}-x_{1} \lambda_{1}+x_{1} c_{1} \mu_{1}+x_{1} a_{12} \mu_{2}+\cdots+x_{1} a_{1 m-1} \mu_{n-1}+x_{1}^{2} c_{1} \mu_{m}, \mu_{m}\right\rangle .
\end{aligned}
$$

Then we have $\theta_{2}\left(\alpha_{i}\right)=\alpha_{i}$ for $1 \leqq i \leqq m-1$, and 


$$
\begin{aligned}
\theta_{2}(\alpha)= & \left(y_{1}+x_{1} c_{1}\right) \mu_{1}+x_{2} \lambda_{2}+\ldots+x_{m-1} \lambda_{m-1} \\
& +\left(y_{m-1}+x_{1} a_{m-1}\right) \mu_{m-1}+\lambda_{m}+w \mu_{m},
\end{aligned}
$$

so that the coefficient of $\lambda_{1}$ is now zero. By repeating this process all the coefficients of $\lambda_{1}, \cdots, \lambda_{m-1}$ may be reduced to zero. But then, using the conditions $\alpha_{i} \cdot \alpha=\alpha_{i} \cdot \alpha_{m}$ for $1 \leqq i \leqq m-1$, and $\alpha^{2}=\alpha_{m}^{2}$, we find that we have succeeded in mapping $\alpha$ into $\alpha_{m}$, while leaving $\alpha_{i}$, $1 \leqq i \leqq m-1$, invariant. This completes the proof of the theorem when $L$ is improper.

3. For the rest of this paper $L$ will be considered to be a proper lattice with zero signature. Thus we have

$$
L=\left\langle\xi_{1}, \rho_{1}\right\rangle \oplus \cdots \oplus\left\langle\xi_{n}, \rho_{n}\right\rangle
$$

where $\xi_{i}^{2}=\xi_{i} \cdot \rho_{i}=1$ and $\rho_{i}^{2}=0$ for $1 \leqq i \leqq n$. By (1) we must have $n \geqq 2$. A primitive vector $\alpha=\sum_{i=1}^{n}\left(a_{i} \xi_{i}+b_{i} \rho_{i}\right)$ is characteristic if and only if $a_{i} \equiv 0(\bmod 2)$ and $b_{i} \equiv 1(\bmod 2)$ for each $i$. (We see this by applying the condition $\alpha \cdot \beta \equiv \beta^{2}(\bmod 2)$ with $\beta$ ranging through the basis vectors $\left.\xi_{i}, \rho_{i}\right)$.

Lemma 2. A primitive vector $\alpha \in L$ may be embedded in a binary sublattice $B$ which splits $L$. If $\alpha$ is characteristic then $B$ is proper and $B^{\perp}$ is improper. If $\alpha$ is not characteristic, then $B$ is proper if $\alpha^{2}$ is odd, and $B$ is improper if $\alpha^{2}$ is even.

Proof. From Wall [1, p. 333], if $\alpha^{2}=2 \alpha+1$ (and hence $\alpha$ is not characteristic), we can map $\alpha$ into $\xi_{1}+a \rho_{1}$. Thus an isometric image of $\alpha$ is contained in $\left\langle\xi_{1}, \rho_{1}\right\rangle$. Apply the inverse isometry to $L$. This will embed $\alpha$ in the inverse image of $\left\langle\xi_{1}, \rho_{1}\right\rangle$. If $\alpha$ is not characteristic and $\alpha^{2}=2 a$, then we may map $\alpha$ into

$$
\beta=(a-1) \rho_{1}+\xi_{1}+\xi_{2} .
$$

Then $\beta \cdot \rho_{2}=1$. Put $\zeta=\beta-a \rho_{2}$, so that $\zeta^{2}=0$ and $\zeta \cdot \rho_{2}=1$. Then $\beta \in H=\left\langle\zeta, \rho_{2}\right\rangle$, a binary sublattice splitting $L$. Thus $\alpha$ may similarly be embedded in an improper binary sublattice which splits $L$.

Finally, we consider the case where $\alpha$ is characteristic with norm $8 b$. Take a splitting of $L$ of the form

$$
L=\langle\xi\rangle \oplus\langle\eta\rangle \oplus H_{2} \oplus \cdots \oplus H_{n}
$$

where $\xi^{2}=-\eta^{2}=1$. The vector $\beta=(2 b+1) \xi+(2 b-1) \eta$ is characteristic with norm $8 b$. Therefore $\alpha$ may be mapped by an isometry into $\beta \in\langle\xi\rangle \oplus\langle\eta\rangle$, and the result follows as before. This completes the proof of the lemma. 
We will now consider the case where $J$ and $K$ do not contain characteristic vectors. We obtain an embedding of an isometric image of $J$ as close as possible to that obtained in $\S 2$. Suppose we have already obtained $\psi(J)=\left\langle\alpha_{1}, \cdots, \alpha_{h}, \beta_{1}, \cdots, \beta_{k}\right\rangle$ where $\alpha_{1}, \cdots, \alpha_{h}$ are of the form given in (3) and thus embedded in a sublattice

$$
L_{h}=\left\langle\lambda_{1}, \mu_{1}\right\rangle \oplus \cdots \oplus\left\langle\lambda_{h}, \mu_{h}\right\rangle
$$

which splits $L$. Assuming that $k \geqq 3$, we now show how to obtain $\alpha_{h+1}$ (and as a special case $\alpha_{1}$, to start the construction).

At least one of the three vectors $\beta_{1}, \beta_{3}, \beta_{1}+\beta_{3}$ must have even norm. We may therefore assume, changing the basis of $\psi(J)$ if necessary, that $\beta_{1}^{2}$ and $\beta_{2}^{2}$ are even. Write

$$
\beta_{i}=\sigma_{i}+d_{i} \tau_{i}, \quad 1 \leqq i \leqq k,
$$

where $\tau_{i} \in L_{h}^{\frac{1}{h}}$ is primitive and $\sigma_{i} \in L_{h}$. It is possible that the $\tau_{i}$, while not characteristic vectors in $L$, may be characteristic vectors in $L_{h}^{+}$. However, replacing $\beta_{1}$ by a linear combination of $\beta_{1}$ and $\beta_{2}$ if necessary, we may assume $\tau_{1}$ at least is not characteristic in $L_{h}^{L}$. (We may achieve this by eliminating a suitable basis vector $\rho$ between $\tau_{1}$ and $\tau_{2}$ ). There are two cases to consider.

Case 1. $\tau_{1}^{2}$ even. Then by Lemma $2, \tau_{1}$ may be embedded in an improper binary sublattice $H_{1}$ of $L_{h}^{\frac{1}{h}}$. Since $k \geqq 2$, we have from (1) that the rank of $\left(L_{h} \oplus H_{1}\right)^{\perp}$ is at least 4 . Therefore, there exists another hyperbolic plane $H_{2}$ such that

$$
L=L_{h} \oplus H_{1} \oplus H_{2} \oplus U \text {. }
$$

But now $\left\langle\alpha_{1}, \cdots, \alpha_{h}, \beta_{1}\right\rangle \subseteq L_{h} \oplus H_{1} \oplus H_{2}$ and we may transform $\beta_{1}$ into the form $\alpha_{h+1}$ using the results already established for improper lattices in $\S 2$.

Case 2. $\tau_{1}^{2}=2 a+1$ odd. Then since $\beta_{1}^{2}$ is even, $d_{1}^{2} \tau_{1}^{2}$ is also even. As in the proof of Lemma 2, $\tau_{1}$ may be embedded in a sublattice $I=\langle\xi, \rho\rangle$ with $\tau_{1}=\xi+a \rho$. Again, from (1), we know the rank of $\left(L_{h} \oplus I\right)^{\perp}$ is at least 4 , so that we may write $L$ in the form

$$
L=L_{h} \oplus I \oplus H \oplus U
$$

where $H=\langle\lambda, \mu\rangle$ is a hyperbolic plane. Adding a linear combination of $\alpha_{1}, \cdots, \alpha_{h}$ to $\beta_{1}$, we may assume $\beta_{1}$ has the form

$$
\beta_{1}=\sum_{i=1}^{h} b_{i} \mu_{i}+d_{1}(\xi+a \rho)
$$

where $\left(b_{1}, \cdots, b_{h}, d_{1}\right)=1$. The next step is to apply isometries to 
$L_{h} \oplus I \oplus H$ that leave $\alpha_{1}, \cdots, \alpha_{h}$ invariant, but change $\beta_{1}$ into a form as above with $d_{1}=1$. As in $\S 2$, we may use $\theta_{1}$ on $L_{h} \oplus H$ and Lemma 2 to achieve this. Applying $\theta_{1}$ on $L_{h} \oplus H$, we transform $\beta_{1}$ into $\beta_{1}+b_{1} \mu$, so that $d_{1} \tau_{1}$ becomes $d_{1} \tau_{1}+b_{1} \mu=d^{\prime} \tau^{\prime}$ (say), where $d^{\prime}=\left(d_{1}, b_{1}\right)$. If now $\tau^{\prime 2}$ is even we use case 1 . Otherwise, as in Lemma 2, we transform $\tau^{\prime}$ into $\xi+a^{\prime} \rho$, and repeat the argument, this time introducing $b_{2} \mu$ by working in $\left\langle\lambda_{1}, \mu_{1}\right\rangle^{\perp}$. Ultimately, since we may reduce $d_{1}$ to 1 , we must get a form with $\tau_{1}^{2}$ even, so that we can use Case 1.

In this manner we may apply a succession of isometries to $J$ until we obtain $\psi(J)=\left\langle\alpha_{1}, \cdots, \alpha_{m-2}, \beta, \gamma\right\rangle$ where $\alpha_{1}, \cdots, \alpha_{m-2}$ are embedded in an improper sublattice $L_{m-2}$ of $L$. Furthermore, we may assume $\beta^{2}$ is even. Write $\beta=\sigma+d \tau$ where $\tau \in L_{m-2}^{\perp}$ is primitive, and $\sigma \in L_{m-2}$. By adding a linear combination of $\alpha_{1}, \cdots, \alpha_{m-2}$ to $\beta$, we may assume

$$
\beta=\sum_{i=1}^{m-2} b_{i} \mu_{i}+d \tau
$$

and since $J$ is primitive, we have $\left(b_{1}, \cdots, b_{m-2}, d\right)=1$. $\tau$ may or may not be a characteristic vector in $L_{m-2}^{\frac{1}{2}}$. We show first how to reduce $d$ to unity. By Lemma $2 \tau$ may be embedded in a binary lattice $B$. Again by (1), the rank of $\left(L_{m-2} \oplus B\right)^{\perp}$ is at least 4 , so that we may write

$$
L=L_{m-2} \oplus B \oplus H \oplus U
$$

where $H=\langle\lambda, \mu\rangle$ is a hyperbolic plane. Using $\theta_{1}$ on $L_{m-2} \oplus H$ and Lemma 2 , we reduce $d$ to 1 as before. Then $\tau^{2}$ is even.

If $\tau$ is not characteristic in $L_{m-2}^{\perp}$ we may use the argument of case 1 above to transform $\beta$ into $\alpha_{m-1}$. Suppose therefore $\tau$ is characteristic in $L_{m-2}^{\perp}$. But we know $\beta$ is not characteristic in $L$. In (8), with $d=1$, it therefore follows that at least one of the coefficients $b_{i}$ must be odd. For if they were all even, $\beta$ would be characteristic in $L$. Say $b_{s}$ is odd. We apply an isometry of type $\theta_{1}$ to

$$
\left\langle\lambda_{s}, \mu_{s}\right\rangle \oplus\left\langle\lambda_{s+1}, \mu_{s+1}\right\rangle \oplus \cdots \oplus\left\langle\lambda_{m-2}, \mu_{m-2}\right\rangle \oplus H .
$$

Then $\theta_{1}\left(\alpha_{i}\right)=\alpha_{i}$ for $1 \leqq i \leqq m-2$, and $\theta_{1}(\beta)=\beta+b_{s} \mu$. Then $\tau$ becomes $\tau+b_{s} \lambda$ which is no longer characteristic in $L_{m-2}^{\perp}$. Therefore $\beta$ may always be transformed into the form $\alpha_{m-1}$ as before.

It therefore suffices to consider the case $J=\left\langle\alpha_{1}, \cdots, \alpha_{m-1}, \gamma\right\rangle$. We treat $K=\varphi(J)$ in a similar manner. Since the norms of the vectors $\varphi\left(\alpha_{1}\right), \cdots, \varphi\left(\alpha_{m-1}\right)$ are even, and they are not characteristic vectors, they may be embedded in an improper sublattice $L_{m-1}^{\prime}$ which splits $L$. Adding hyperbolic planes to $L_{m-1}$ and $L_{m-1}^{\prime}$ (they exist 
since the rank of $L_{m-1}^{\frac{1}{}}$ is at least 4) and applying our theorem, already established for the improper case, we may assume $\varphi\left(\alpha_{i}\right)=\alpha_{i}$ for $1 \leqq i \leqq m-1$. Thus it suffices to consider $K$ of the form $\left\langle\alpha_{1}, \cdots, \alpha_{m-1}, \delta\right\rangle$. There are now two cases depending on whether $\gamma^{2}=\delta^{2}$ is odd or even.

Case 1. $\gamma^{2}=\delta^{2}$ odd. Using Lemma 2 and $\alpha_{1}, \cdots, \alpha_{m-1}$ to eliminate the coefficients of $\lambda_{1}, \cdots, \lambda_{m-1}, \gamma$ may be written as

$$
\gamma=\sum_{i=1}^{m-1} u_{i} \mu_{i}+d\left(\xi^{\prime}+a \rho^{\prime}\right)
$$

where $\left(u_{1}, \cdots, u_{m-1}, d\right)=1$. $L$ may be split thus

$$
L=L_{m-1} \oplus\left\langle\xi^{\prime}, \rho^{\prime}\right\rangle \oplus\langle\xi, \rho\rangle \oplus U \text {. }
$$

We show first how to reduce $d$ to unity. Apply the isometry

$$
\begin{aligned}
\theta_{3}: & \left\langle\lambda_{1}, \mu_{1}\right\rangle \oplus\left\langle\lambda_{2}, \mu_{2}\right\rangle \oplus \cdots \oplus\left\langle\lambda_{m-1}, \mu_{m-1}\right\rangle \oplus\langle\xi, \rho\rangle \rightarrow \\
& \left\langle\lambda_{1}-c_{1} \rho, \mu_{1}+\rho\right\rangle \oplus\left\langle\lambda_{2}-a_{12} \rho, \mu_{2}\right\rangle \oplus \cdots \oplus\left\langle\lambda_{m-1}-a_{1 m-1} \rho, \mu_{m-1}\right\rangle \\
& \oplus\left\langle\xi-\lambda_{1}+c_{1} \mu_{1}+a_{12} \mu_{2}+\cdots+a_{1 m-1} \mu_{m-1}+c_{1} \rho, \rho\right\rangle .
\end{aligned}
$$

We may easily check that $\theta_{3}\left(\alpha_{i}\right)=\alpha_{i}$ for $1 \leqq i \leqq m-1$. Furthermore $\theta_{3}(\gamma)=\gamma+u_{1} \rho$. Mapping $d\left(\xi^{\prime}+a \rho^{\prime}\right)+u_{1} \rho$ back into $\left\langle\xi^{\prime}, \rho^{\prime}\right\rangle$ we may restore $\gamma$ to the form (9), but now with $d$ dividing $u_{1}$. Now repeating this process in $\left\langle\lambda_{1}, \mu_{1}\right\rangle^{\perp}$, we may obtain a new $\gamma$ with $d$ also dividing $u_{2}$. Since $\left(u_{1}, \cdots, u_{m-1}, d\right)=1$ we ultimately reach a form with $d=1$.

Using again Lemma 2, we may arrange for $\delta$ to have the form

$$
\delta=\sum_{i=1}^{m-1}\left(x_{i} \lambda_{i}+y_{i} \mu_{i}\right)+f\left(\xi^{\prime}+e \rho^{\prime}\right) .
$$

We may assume $\delta-\sum_{i=1}^{m-1} x_{i} \alpha_{i}$ is primitive (since $K$ is primitive) and therefore, using the notation of (7)

$$
\left(f, z_{1}, z_{2}, \cdots, z_{m-1}\right)=1 \text {. }
$$

Applying $\theta_{3}$, we find $\theta_{3}(\delta)=\delta+z_{1} \rho$. By the usual chain of arguments we may assume $f=1$ in (10).

Finally we apply isometries that reduce $x_{1}, \cdots, x_{m-1}$ in turn to zero. Define

$$
\begin{aligned}
& \theta_{4}\left\langle\lambda_{1}, \mu_{1}\right\rangle \oplus\left\langle\lambda_{2}, \mu_{2}\right\rangle \oplus \cdots \oplus\left\langle\lambda_{m-1}, \mu_{m-1}\right\rangle \\
& \quad \oplus\left\langle\xi^{\prime}, \rho^{\prime}\right\rangle \rightarrow\left\langle\lambda_{1}-c_{1} x_{1} \rho^{\prime}, \mu_{1}+x_{1} \rho^{\prime}\right\rangle \\
& \quad \bigoplus\left\langle\lambda_{2}-x_{1} a_{12} \rho^{\prime}, \mu_{2}\right\rangle \oplus \cdots \oplus\left\langle\lambda_{m-1}-x_{1} a_{1 m-1} \rho^{\prime}, \mu_{m-1}\right\rangle \\
& \oplus\left\langle\xi^{\prime}-x_{1} \lambda_{1}+x_{1} c_{1} \mu_{1}+x_{1} a_{12} \mu_{2}+\cdots+x_{1} a_{1 m-1} \mu_{m-1}+x_{1}^{2} c_{1} \rho^{\prime}, \rho^{\prime}\right\rangle .
\end{aligned}
$$

Then $\theta_{4}\left(\alpha_{i}\right)=\alpha_{i}$ for $1 \leqq i \leqq m-1$, and 


$$
\begin{aligned}
\theta_{4}(\delta)= & \left(y_{1}+x_{1} c_{1}\right) \mu_{1}+x_{2} \lambda_{2}+\cdots+x_{m-1} \lambda_{m-1} \\
& +\left(y_{m-1}+x_{1} a_{1 m-1}\right) \mu_{m-1}+\xi^{\prime}+e^{\prime} \rho^{\prime} .
\end{aligned}
$$

We have thus reduced the coefficient of $\lambda_{1}$ to zero. Proceeding in this manner we may reduce all the coefficients of $\lambda_{1}, \cdots, \lambda_{m-1}$ to zero. Using the conditions $\alpha_{i} \cdot \gamma=\alpha_{i} \cdot \delta$ and $\gamma^{2}=\delta^{2}$, we find we have mapped $\delta$ into $\gamma$, and hence $K$ into $J$, by an isometry of $L$. This completes the proof in this case.

Case 2. $\gamma^{2}=\delta^{2}$ even. Write $\gamma=\sigma+d \tau$ where $\tau \in L_{m-1}^{\perp}$ is primitive and $\sigma \in L_{m-1}$. We first show that we may take $d=1$. We use a combination of the previous methods. We may assume $\gamma$ has the form (compare (8))

$$
\gamma=\sum_{i=1}^{m-1} u_{i} \mu_{i}+d \tau
$$

where $\left(u_{1}, \cdots, u_{m-1}, d\right)=1$. If $\tau$ is characteristic in $L_{m-1}^{\frac{1}{m}}$, we may embed $\tau$ in a proper binary lattice $B$ such that

$$
L_{m-1}^{\perp}=B \oplus H_{1} \oplus \cdots \oplus H_{t} .
$$

Applying the isometry $\theta_{1}$ on $L_{m-1} \oplus H_{1}$, as before, we may assume $d$ divides $u_{1}$. If $\tau$ is not characteristic in $L_{m-1}^{+}$, we embed $\tau$ in a binary lattice $B$ so that $L$ splits thus

$$
L=L_{m-1} \oplus B \oplus\langle\xi, \rho\rangle \oplus U \text {. }
$$

Applying $\theta_{3}$ on $L_{m-1} \oplus\langle\xi, \rho\rangle$, as before, we may assume $d$ divides $u_{1}$. Proceeding in this manner we reduce $d$ to unity. Then $\tau^{2}$ is even and may be embedded in a hyperbolic plane $H$ (after another isometry if $\tau$ is characteristic in $L_{m-1}^{\perp}$ ), so that, in fact, $\gamma$ takes the form $\alpha_{m}$ given in (3).

By similar reasoning $\delta$ may be written

$$
\delta=\sum_{i=1}^{m-1}\left(x_{i} \lambda_{i}+y_{i} \mu_{i}\right)+d \tau,
$$

$d$ reduced to unity, and $\tau$ embedded in $H$. Finally we reduce the coefficients $x_{1}, \cdots, x_{m-1}$ to zero by applying $\theta_{2}$, exactly as at the end of $\S 2$.

This completes the proof of the theorem when $J$ and $K$ contain no characteristic vectors.

4. It remains for us to consider the case where $J$ and $K$ contain characteristic vectors. As in $\S 3, L$ has the form

$$
L=\left\langle\xi_{1}, \rho_{1}\right\rangle \oplus \cdots \oplus\left\langle\xi_{n}, \rho_{n}\right\rangle
$$


where $\xi_{i}^{2}=\xi_{i} \cdot \rho_{i}=1$ and $\rho_{i}^{2}=0,1 \leqq i \leqq n$.

We may choose a basis for $J$ that contains only one characteristic vector; for example, eliminate the coefficients of $\rho_{1}$ in all but one of the basis vectors. Applying the results of the previous section, it therefore suffices to consider the special case where

$$
J=\left\langle\alpha_{1}, \cdots, \alpha_{m-2}, \beta, \gamma\right\rangle \text { and } K=\left\langle\alpha_{1}, \cdots, \alpha_{m-2}, \beta, \delta\right\rangle
$$

with the $\alpha_{i}$ as in (3), $\delta=\varphi(\gamma)$ is characteristic, and with $\beta$ either $\alpha_{m-1}$ (if $\beta^{2}$ is even) or of the form given in (9) with $d=1$. There are therefore two cases to consider depending on whether the norm of $\beta$ is even or odd.

Case 1. $\beta^{2}$ even; so that $\beta=\alpha_{m-1}$ and $J=\left\langle\alpha_{1}, \cdots, \alpha_{m-1}, \gamma\right\rangle . \quad \gamma$ may be assumed to have the form

$$
\gamma=\sum_{i=1}^{m-1} u_{i} \mu_{i}+d(2 \xi+(2 e-1) \rho)
$$

(after using the $\alpha_{i}$ to eliminate the coefficients of the $\lambda_{i}$, and Lemma 2 to simplify the component of $\gamma$ in $\left.L_{m-1}^{\perp}\right)$. $L$ may now be written

$$
L=L_{m-1} \oplus\langle\xi, \rho\rangle \oplus U
$$

where $U$ is an orthogonal sum of hyperbolic planes. By the usual argument we may reduce $d$ to unity. Similarly, we can transform $\delta$ into

$$
\delta=\sum_{i=1}^{m-1}\left(x_{i} \lambda_{i}+y_{i} \mu_{i}\right)+2 \xi+(2 f-1) \rho .
$$

It therefore remains to transform $\delta$ into a form where the coefficients of $\lambda_{i}$ are zero. Since $\delta$ is characteristic

$$
x_{i}=\delta \cdot \mu_{i} \equiv \mu_{i}^{2} \equiv 0(\bmod 2), \quad 1 \leqq i \leqq m-1 .
$$

Now apply the isometry

$$
\begin{aligned}
& \theta_{5}:\left\langle\lambda_{1}, \mu_{1}\right\rangle \oplus\left\langle\lambda_{2}, \mu_{2}\right\rangle \oplus \cdots \oplus\left\langle\lambda_{m-1}, \mu_{m-1}\right\rangle \\
& \oplus\langle\xi, \rho\rangle \rightarrow\left\langle\lambda_{1}-\frac{1}{2} x_{1} c_{1} \rho, \mu_{1}+\frac{1}{2} x_{1} \rho\right\rangle \\
& \oplus\left\langle\lambda_{2}-\frac{1}{2} x_{1} a_{12} \rho, \mu_{2}\right\rangle \oplus \cdots \oplus\left\langle\lambda_{m-1}-\frac{1}{2} x_{1} a_{1 m-1} \rho, \mu_{m-1}\right\rangle \\
& \oplus\left\langle\xi-\frac{1}{2} x_{1} \lambda_{1}+\frac{1}{2} x_{1} c_{1} \mu_{1}+\frac{1}{2} x_{1} a_{12} \mu_{2}+\cdots\right. \\
&\left.+\frac{1}{2} x_{1} a_{1 m-1} \mu_{m-1}+\frac{1}{4} x_{1}^{2} c_{1} \rho, \rho\right\rangle .
\end{aligned}
$$

Then $\theta_{5}\left(\alpha_{i}\right)=\alpha_{i}$ for $1 \leqq i \leqq m-1$, and 


$$
\begin{aligned}
\theta_{5}(\delta)= & \left(y_{1}+x_{1} c_{1}\right) \mu_{1}+x_{2} \lambda_{2}+\cdots+x_{m-1} \lambda_{m-1} \\
& +\left(y_{m-1}+x_{1} a_{1 m-1}\right) \mu_{m-1}+2 \xi+\left(2 f^{\prime}-1\right) \rho .
\end{aligned}
$$

We have thus reduced the coefficient of $\lambda_{1}$ to zero. Proceeding in this manner, we may reduce all the coefficients of the $\lambda_{i}$ in turn to zero. Finally, since $\alpha_{i} \cdot \gamma=\alpha_{i} \cdot \delta$ and $\gamma^{2}=\delta^{2}$, the coefficients of $\delta$ now match those in $\gamma$, so that we have mapped $\delta$ into $\gamma$, and so $K$ into $J$. This completes the proof in this case.

Case 2. $\beta^{2}$ odd. Then $\beta$ may be chosen as

$$
\beta=\sum_{i=1}^{m-2} b_{i} \mu_{i}+\xi+b \rho .
$$

Using $\alpha_{1}, \cdots, \alpha_{m-2}$ and $\beta$ to eliminate the coefficients of $\lambda_{1}, \cdots, \lambda_{m-2}$ and $\xi$, we may write $\gamma$ as

$$
\gamma=\sum_{i=1}^{m-2} u_{i} \mu_{i}+u \rho+d\left(2 \xi^{\prime}+(2 e-1) \rho^{\prime}\right) .
$$

$L$ is now split into the form

$$
L=L_{m-2} \oplus\langle\xi, \rho\rangle \oplus\left\langle\xi^{\prime}, \rho^{\prime}\right\rangle \oplus H \oplus U
$$

where $H=\langle\lambda, \mu\rangle$ and $U$ is an improper lattice (see Lemma 2). We now reduce the coefficient $d$ to unity. Isometries on $L_{m-2} \oplus\langle\xi, \rho\rangle \oplus H$ of the type

$$
\begin{aligned}
\theta_{6}: & \left\langle\lambda_{1}, \mu_{1}\right\rangle \oplus\left\langle\lambda_{2}, \mu_{2}\right\rangle \oplus \cdots \oplus\left\langle\lambda_{m-2}, \mu_{m-2}\right\rangle \oplus\langle\xi, \rho\rangle \\
& \oplus\langle\lambda, \mu\rangle \rightarrow\left\langle\lambda_{1}-c_{1} \mu, \mu_{1}+\mu\right\rangle \oplus\left\langle\lambda_{2}-a_{12} \mu, \mu_{2}\right\rangle \oplus \cdots \\
& \oplus\left\langle\lambda_{m-2}-a_{1 m-2} \mu, \mu_{m-2}\right\rangle \oplus\left\langle\xi-b^{1} \mu, \rho\right\rangle \\
& \oplus\left\langle\lambda-\lambda_{1}+c_{1} \mu_{1}+a_{12} \mu_{2}+\cdots+a_{1 m-2} \mu_{m-2}+b_{1} \rho+c_{1} \mu, \mu\right\rangle
\end{aligned}
$$

leave $\alpha_{1}, \cdots, \alpha_{m-2}$ and $\beta$ invariant. $\gamma$ is transformed into $\gamma+u_{1} \mu$, so that with the usual argument we may assume $d$ divides $u_{1}$. We may transform $\gamma$ in this manner into a form where $(u, d)=1$.

Since $\gamma$ is characteristic we know $\gamma \cdot \xi^{\prime} \equiv 1(\bmod 2)$, and hence that $d$ is odd. Now apply the isometry

$$
\begin{aligned}
& \theta_{7}:\langle\xi, \rho\rangle \oplus\langle\lambda, \mu\rangle \rightarrow\langle\xi-2 b \mu, \rho+2 \mu\rangle \\
& \oplus\langle\lambda-2 \xi+2(1+b) \rho+2(2 b+1) \mu, \mu\rangle .
\end{aligned}
$$

This leaves $\alpha_{1}, \cdots, \alpha_{m-2}$ and $\beta$ invariant and transforms $\gamma$ into $\gamma+2 u \mu$. Since $(2 u, d)=1$, we may reduce $d$ to 1 in $\gamma$.

As above we may also put $\delta=\varphi(\gamma)$ into the form

$$
\delta=\sum_{i=1}^{m-2}\left(x_{i} \lambda_{i}+y_{i} \mu_{i}\right)+v \xi+w \rho+2 \xi^{\prime}+(2 f-1) \rho^{\prime} .
$$


Since $\delta$ is characteristic, we have $x_{i} \equiv y_{i} \equiv 0(\bmod 2)$ for each $i$, $v \equiv 0(\bmod 2)$ and $w \equiv 1(\bmod 2)$.

It now remains to reduce the coefficients $x_{1}, \cdots, x_{m-2}, v$ to zero. First apply the isometry

$$
\begin{aligned}
\theta_{8}:\left\langle\lambda_{1}, \mu_{1}\right\rangle \oplus\left\langle\lambda_{2}, \mu_{2}\right\rangle \oplus \cdots \oplus\left\langle\lambda_{m-2}, \mu_{m-2}\right\rangle \oplus\langle\xi, \rho\rangle \oplus\left\langle\xi^{\prime}, \rho^{\prime}\right\rangle \rightarrow \\
\left\langle\lambda_{1}-\frac{1}{2} x_{1} c_{1} \rho^{\prime}, \mu_{1}+\frac{1}{2} x_{1} \rho^{\prime}\right\rangle \oplus\left\langle\lambda_{2}-\frac{1}{2} x_{1} a_{12} \rho^{\prime}, \mu_{2}\right\rangle \oplus \cdots \\
\oplus\left\langle\lambda_{m-2}-\frac{1}{2} x_{1} a_{1 m-2} \rho^{\prime}, \mu_{m-2}\right\rangle \oplus\left\langle\xi-\frac{1}{2} x_{1} b_{1} \rho^{\prime}, \rho\right\rangle \\
\oplus\left\langle\xi^{\prime}-\frac{1}{2} x_{1} \lambda_{1}+\frac{1}{2} x_{1} c_{1} \mu_{1}+\frac{1}{2} x_{1} a_{12} \mu_{2}+\cdots\right. \\
\left.+\frac{1}{2} x_{1} a_{1 m-2} \mu_{m-2}+\frac{1}{2} x_{1} b_{1} \rho+\frac{1}{4} x_{1}^{2} c_{1} \rho^{\prime}, \rho^{\prime}\right\rangle .
\end{aligned}
$$

Then $\theta_{8}\left(\alpha_{i}\right)=\alpha_{i}$ for $1 \leqq i \leqq m-2, \theta_{8}(\beta)=\beta$, and in $\theta_{8}(\delta)$ the coefficient of $\lambda_{1}$ is zero. Working now in $\left\langle\lambda_{1}, \mu_{1}\right\rangle^{\perp}$ we reduce the coefcient of $\lambda_{2}$ to zero. We may therefore assume

$$
x_{1}=x_{2}=\cdots=x_{m-2}=0 \text {. }
$$

The final step, the reduction of $v$ to zero appears to be more difficult. If $v \equiv 0(\bmod 4)$ we may apply the isometry

$$
\begin{aligned}
& \theta_{9}:\langle\xi, \rho\rangle \oplus\left\langle\xi^{\prime}, \rho^{\prime}\right\rangle \rightarrow\left\langle\xi-\frac{1}{2} v b \rho^{\prime}, \rho+\frac{1}{2} v \rho^{\prime}\right\rangle \\
& \oplus\left\langle\xi^{\prime}-\frac{1}{2} v \xi+\frac{1}{2} v(1+b) \rho+t \rho^{\prime}, \rho^{\prime}\right\rangle
\end{aligned}
$$

where $2 t=(1 / 4) v^{2}(1+2 b)$. (If $v \equiv 2(\bmod 4)$ then $\left.t \notin Z\right)$. Then $\theta_{9}$ leaves $\alpha_{1}, \cdots, \alpha_{m-2}$ and $\beta$ invariant, while the coefficient of $\xi$ in $\theta_{9}(\delta)$ is reduced to zero. From the various products $\delta \cdot \alpha_{i}=\gamma \cdot \alpha_{i}$, $1 \leqq i \leqq m-2, \delta \cdot \beta=\gamma \cdot \beta$ and $\delta^{2}=\gamma^{2}$ we see that all the coefficients of $\delta$ (actually an isometric image of our original $\delta$ ) now match those of $\gamma$. Thus we have mapped $\delta$ into $\gamma$ and so $K$ into $J$.

If, however, $v \equiv 2(\bmod 4)$ we must modify the above argument. We first change the basis of $L$ so that $G=\left\langle\xi^{\prime}, \rho^{\prime}\right\rangle \oplus\langle\lambda, \mu\rangle$ becomes $G=\left\langle\xi_{1}, \rho_{1}\right\rangle \oplus\left\langle\xi_{2}, \rho_{2}\right\rangle$ where $\xi_{i}^{2}=\xi_{i} \cdot \rho_{i}=1$ and $\rho_{i}^{2}=0$ for $i=1,2$. Since the characteristic vector $2 \xi^{\prime}+(2 f-1) \rho^{\prime}$ in $G$ can be mapped into any other characteristic vector of $G$ by an isometry, we may assume $\delta$ has the form

$$
\delta=\sum_{i=1}^{m-2} y_{i} \ell_{i}+v \xi+w \rho+2 \xi_{1}+\left(2 e_{1}-1\right) \rho_{1}+2 \xi_{2}+\left(2 e_{2}-1\right) \rho_{2}
$$

where $e_{1}$ is chosen such that 


$$
2 e_{1}+w-1 \equiv 0(\bmod 4),
$$

(recall that $w \equiv 1(\bmod 2)$ since $\delta$ is characteristic).

We now apply the isometry

$$
\begin{aligned}
& \theta_{10}:\langle\xi, \rho\rangle \oplus\langle\left\langle\xi_{1}, \rho_{1}\right\rangle \rightarrow \\
&\left\langle(1-b) \xi+b(1+b) \rho+b \xi_{1}+b(b-1) \rho_{1},\right. \\
&\left.\xi-b \rho-\xi_{1}+(1-b) \rho_{1}\right\rangle \\
& \oplus\left\langle-b \xi+b(1+b) \rho+(1+b) \xi_{1}+b(b-1) \rho_{1},\right. \\
&\left.-\xi+(1+b) \rho+\xi_{1}+b \rho_{1}\right\rangle .
\end{aligned}
$$

Again $\alpha_{1}, \cdots, \alpha_{m-2}$ and $\beta$ are left invariant by $\theta_{10}$. But the coefficient of $\xi$ is changed from $v$ to $v^{\prime}=v-v b+w-2 b-\left(2 e_{1}-1\right)$. But now

$$
\begin{aligned}
v^{\prime} & \equiv 2-2 b+w-2 b-2 e_{1}+1 \\
& \equiv 2 e_{1}-1+w \equiv 0(\bmod 4) .
\end{aligned}
$$

After restoring $G$ to the form $\left\langle\xi^{\prime}, \rho^{\prime}\right\rangle \oplus\langle\lambda, \mu\rangle$ we are in a position to finish the proof by means of the isometry $\theta_{9}$ as above.

\section{REFERENCE}

1. C. T. C. Wall, On the orthogonal groups of unimodular quadratic forms, Math. Ann. 147 (1962), 328-338.

Received August 23, 1967. This research was partially supported by the National Science Foundation through grant GP-6663.

The Pennsylvania State University 


\title{
PACIFIC JOURNAL OF MATHEMATICS
}

\author{
EDITORS
}

\author{
H. ROYDEN \\ Stanford University \\ Stanford, California
}

\author{
J. P. JANS \\ University of Washington \\ Seattle, Washington 98105
}

\section{J. DUGUNDJI}

Department of Mathematics University of Southern California Los Angeles, California 90007

\section{RICHARD ARENS}

University of California Los Angeles, California 90024

\section{ASSOCIATE EDITORS}

\author{
E. F. BECKENBACH
}

B. H. NEUMANN

F. WoLF

K. YosidA

\section{SUPPORTING INSTITUTIONS}

\author{
UNIVERSITY OF BRITISH COLUMBIA \\ CALIFORNIA INSTITUTE OF TECHNOLOGY \\ UNIVERSITY OF CALIFORNIA \\ MONTANA STATE UNIVERSITY \\ UNIVERSITY OF NEVADA \\ NEW MEXICO STATE UNIVERSITY \\ OREGON STATE UNIVERSITY \\ UNIVERSITY OF OREGON \\ OSAKA UNIVERSITY \\ UNIVERSITY OF SOUTHERN CALIFORNIA
}

\author{
STANFORD UNIVERSITY \\ UNIVERSITY OF TOKYO \\ UNIVERSITY OF UTAH \\ WASHINGTON STATE UNIVERSITY \\ UNIVERSITY OF WASHINGTON \\ AMERICAN MATHEMATICAL SOCIETY \\ CHEVRON RESEARCH CORPORATION \\ TRW SYSTEMS \\ NAVAL WEAPONS CENTER
}

Mathematical papers intended for publication in the Pacific Journal of Mathematics should be in typed form or offset-reproduced, double spaced with large margins. Underline Greek letters in red, German in green, and script in blue. The first paragraph or two must be capable of being used separately as a synopsis of the entire paper. It should not contain references to the bibliography. Manuscripts, in duplicate if possible, may be sent to any one of the four editors. All other communications to the editors should be addressed to the managing editor, Richard Arens, University of California, Los Angeles, California 90024.

Each author of each article receives 50 reprints free of charge; additional copies may be obtained at cost in multiples of 50 .

The Pacific Journal of Mathematics is published monthly. Effective with Volume 16 the price per volume (3 numbers) is $\$ 8.00$; single issues, $\$ 3.00$. Special price for current issues to individual faculty members of supporting institutions and to individual members of the American Mathematical Society: $\$ 4.00$ per volume; single issues $\$ 1.50$. Back numbers are available.

Subscriptions, orders for back numbers, and changes of address should be sent to Pacific Journal of Mathematics, 103 Highland Boulevard, Berkeley 8, California.

Printed at Kokusai Bunken Insatsusha (International Academic Printing Co., Ltd.), 7-17, Fujimi 2-chome, Chiyoda-ku, Tokyo, Japan.

PUBLISHED BY PACIFIC JOURNAL OF MATHEMATICS, A NON-PROFIT CORPORATION

The Supporting Institutions listed above contribute to the cost of publication of this Journal, but they are not owners of publishers and have no responsibility for its content or policies. 


\section{Pacific Journal of Mathematics}

Vol. 26, No. 2 December, 1968

Seymour Bachmuth and Horace Yomishi Mochizuki, Kostrikin's theorem on

Engel groups of prime power exponent ....................

Paul Richard Beesack and Krishna M. Das, Extensions of Opial's inequality ...................................... 215

John H. E. Cohn, Some quartic Diophantine equations .............. 233

H. P. Dikshit, Absolute $(C, 1) \cdot\left(N, p_{n}\right)$ summability of a Fourier series and its conjugate series ............................... 245

Raouf Doss, On measures with small transforms ................. 257

Charles L. Fefferman, $L_{p}$ spaces over finitely additive measures........ 265

Le Baron O. Ferguson, Uniform approximation by polynomials with integral coefficients. II...................................

Takashi Ito and Thomas I. Seidman, Bounded generators of linear

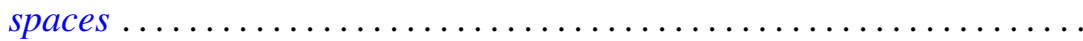

Masako Izumi and Shin-ichi Izumi, Nörlund summability of Fourier series ..........................................

Donald Gordon James, On Witt's theorem for unimodular quadratic

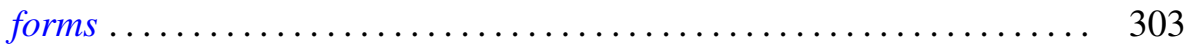

J. L. Kelley and Edwin Spanier, Euler characteristics .............. 317

Carl W. Kohls and Lawrence James Lardy, Some ring extensions with matrix

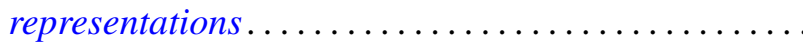

Ray Mines, III, A family of functors defined on generalized primary groups ....

Louise Arakelian Raphael, A characterization of integral operators on the space of Borel measurable functions bounded with respect to a weight function....

Charles Albert Ryavec, The addition of residue classes modulo $n .$.

H. M. (Hari Mohan) Srivastava, Fractional integration and inversion formulae associated with the generalized Whittaker transform ...

Edgar Lee Stout, The second Cousin problem with bounded data ...

Donald Curtis Taylor, A generalized Fatou theorem for Banach

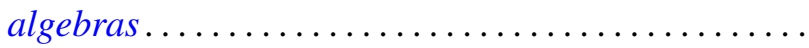

Bui An Ton, Boundary value problems for elliptic convolution equations of Wiener-Hopf type in a bounded region...

Philip C. Tonne, Bounded series and Hausdorff matrices for absolutely convergent sequences... 